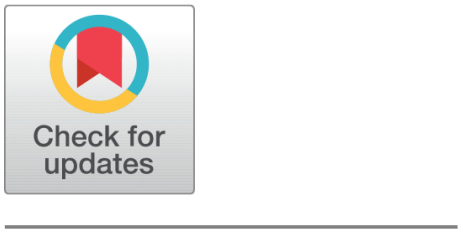

OPEN ACCESS

Received: 15.12.2019

Accepted: 23.12 .2019

Published: 05.01.2020

Editor: Dr. D. R. Galfat

Citation: Sarad R, Aggarwal R, Singh B, Singh D (2020) Comparative study of cruciate retaining vs posterior stabilized prosthesis in total knee replacement. International Journal of Orthopaedics Traumatology \& Surgical Sciences 6(1): 75-80. https:// doi.org/10.47618/IJOTSS/v6i1.15

* Corresponding author.

Funding: None

Competing Interests: None

Copyright: (c) 2020 Sarad et al. This is an open access article distributed under the terms of the Creative Commons Attribution License, which permits unrestricted use, distribution, and reproduction in any medium, provided the original author and source are credited.

Published By Society of Orthopaedics, Surgical and Dental Sciences

ISSN

Print: 2455-0809

Electronic: 2454-4167

\title{
Comparative study of cruciate retaining vs posterior stabilized prosthesis in total knee replacement
}

\author{
Rajan Sarad ${ }^{1}$, Rajkumar Aggarwal ${ }^{1}$, Baljit Singh ${ }^{1}$, Devinderpal Singh ${ }^{1 *}$ \\ 1 Department of orthopaedics, Sri Guru Ramdas university of Health Sciences, Amritsar, \\ Punjab, India
}

\begin{abstract}
Introduction: Although being debated for many years, the superiority of Posterior Cruciate-Retaining (CR) Total knee Arthroplasty (TKA) and Posterior Cruciate - Stabilizing (PS) TKA remains controversial. The primary objective of this study was to compare functional outcome in patients who underwent primary total knee arthroplasty surgery using posterior stabilized and cruciate retaining implants. Material and method: Sixty diseased osteoarthritis knees in 42 patients were included in the study, with 30 cases (Knees) operated with CR prosthesis and 30 cases (Knees) with PS Prosthesis. The two groups were then compared using disease specific functional scores; 2011 knee society score. Pain, range of motion, any flexion, varus, valgus deformity, instability at knee joint was assessed preroperatively and postoperatively. Functional outcome (e.g. walking, climbing stairs) was assessed. Result: Postperative objective knee score and range of motion was significantly better in PS group than CR group, with significant $\mathrm{p}$ value. No significant difference in functional outcome between both the groups was seen. Conclusion: No significant difference in the functional outcome is seen between both the groups.
\end{abstract}

Keywords: Posterior cruciate stabilizing; posterior cruciate retaining; total knee arthroplasty

\section{Introduction}

Total Knee arthroplasty, a surgical procedure to replace weight-bearing surfaces of knee joint to relieve pain and disability; is an end-of-line treatment for patients with severe pain and functional limitations. Although being debated for many years, the superiority of Posterior CruciateRetaining (CR) Total knee Arthroplasty (TKA) and Posterior Cruciate-Stabilizing (PS) TKA remains controversial. Proponents of Cruciate-Retaining; CR pros- thesis assert that PCL retention preserves more normal knee kinematics ${ }^{(1)}$ , improved proprioception ${ }^{(2,3)}$, superior Knee stability (PCL preventing anterior translation of the femur on the tibia) and is more of bone conserving Procedure whereas the proponents of PCL Stabilized; PS prosthesis assert that Posteriorstabilized implants with a polyethylene post and femoral cam to replace the role of the PCL, has controlled replication of the femoral rollback 
mechanism, resulting in reduced sliding sheer stress on the polyethylene liner and providing superior range of motion ${ }^{(4)}$ in terminal knee flexion, a less technically demanding procedure, a more stable component interface,

Also PCL is diseased with various form of arthritis and contracture and is difficult to balance reproducibly ${ }^{(5)}$, so the significant deformity can be more reliably corrected with the use of PCL substituting designs. Multiple studies have addressed differences in knee range of motion following cruciate retaining and posterior stabilized primary knee replacements. Most studies have shown a small gain in terminal flexion with posterior stabilized designs. ${ }^{(6-8)}$ A number of studies have also reported on short- and medium-term functional results comparing these two implant designs. The current literature has not demonstrated any significant difference in early functional outcome between posterior stabilized andcruciate retaining knee replacements. This discrepancybetweenbiomechanical and clinical results suggests that the differences in function between PS and CR knees are either nonexistent or that they are not detected with current outcome tools. The primary objective of this study was to compare functional outcome in patients who underwent primary total knee arthroplasty surgery using posteriorstabilized and cruciate retaining implants.

\section{Material And Method}

Patient in age group 55 to 70 years with diseased osteoarthritic knees undergoing either unilateral or bilateral primary Total knee arthroplsty at our centre were selected for the study. The prospective study was conducted on 60 diseased osteoarthritis knee in 42 patients operated either with CR or PS Total knee arthroplasty for duration of One and half years from Dec 17 to May 19 with prospective analysis upto Oct. 2019 with follow up for period of 6 months. Patients with age group 55 to 70 years, Osteoarthritis radiological grade III\&IV, painful knees with inability to perform routine activities and willing to giving consent for both CR and PS were included in the study; whereas patients with sepsis of the knee joint or inflammatory arthritis, Patients with local skin lesions, Revision Total knee arthroplasty and Patients with prior PCL injury were excluded From the study. The two groups were then compared using disease specific functional scores; 2011 knee society score. Pain, range of motion, any flexion, varus, valgus deformity, instability at knee joint was assessed preroperatively and postoperatively. Functional outcome(e.g.alking, climbing stairs) was assessed. Patient expectation with the Total knee arthroplasty procedure and satisfaction was noted. The criteria were assessed preoperatively and postoperatively and further at follow up in the OPD at $4^{\text {th }}, 8^{\text {th }}, 12^{\text {th }}$ and $24^{\text {th }}$ week and points were assigned as per " 2011 knee society score" and the results were compared for cruciate retaining andposterior stabilised prosthesis. Patients were operated in standard arthroplasty operative setup. All Surgeries done using midline anterior skin incision followed by medial parapatellar arthrotomy. Femoral and tibial cuts taken with help of intra-medullary and extra-medullary jig respectively. In CR group, we tried to keep tibial slope as normal as possible while in PS group we tried to keep tibial slope $5^{\circ} \pm 1^{\circ}\left(3^{\circ}\right.$ cutting tibial cutting block was used). Negative suction drain was kept for 24 hours in all patients. Full weight bearing walking with support started from 1st post- op day and stair climbing was allowed from 2nd post-op day. All patients were discharged on 3rd or 4th post op day. Physiotherapy was advised for 23 weeks. Stitches were removed on 18th post-op day. After that support gradually weaned off over period of 2 weeks. All patients re-evaluated clinically, radiologically (as it was done pre-operatively) and for complications for the period of 6 months.

\section{Results}

\subsection{Baseline Demographics}

The mean age of the patients in the CR group was 61.53 years and for PS group was 62.03 years. Out of 30 knees Operated with CR knees, 25 knees were of female gender and 5 were of male gender. Similarly, for PS knees; 13 knees were Of female and 17 were of male. 12 patients were operated with U/L CR arthroplasty and 7 patients with unilateral CR arthroplasty and 7 patients with B/L CR arthroplasty 7 patients were operated with B/L PS arthroplastyand 12 with U/L PS arthroplasty .4 patients were mixed, treated with CR arthroplasty in one knee and PS in the other.

\subsection{Primary Outcome}

The mean objective knee score of CR group improved from 33.17 to 71.17 and that for PS group, from 39.1 to 73.13 postoperatively; with $\mathrm{p}$ value of 0.018 which indicated significant difference in favour of PS group.

The mean range of motion of CR Group preoperatively was 87.5 degree which got improved to 104.2 degree postoperatively and from 92.33 degree to 114.67 degree for the PS group with significant difference between both the group postoperatively with $\mathrm{p}$ value of 0.010 in favour of PS group were $33.63 \& 69.23$ respectively, compared to mean preoperative post-operative functional score of PS group of 31.33 \& 66.37), indicated insignificant difference with $p$ value of 0.101 . The Postoperative symptoms score in CR group was mean 7 . 23 and that in PS group was 7.03; with p value of 0.654 which indicated insignificant difference between both the groups. The post operative mean satisfaction score was 21.6 in CR group 21.6 in PS group and postoperative mean expectation score was 9.93 in CR group and 9.57 for PS group with both the groups were comparable with insignificant 
Table 1. Preoperative knee society score

\begin{tabular}{llll}
\hline The 2011 KSS categories & CR & PS & P Value \\
\hline Objective Knee Indicators & $33.17 \pm 4.12$ & $39.1 \pm 12.3$ & 0.372 \\
Satisfaction & $7.6 \pm 2.11$ & $7.07 \pm 2.02$ & 1.000 \\
Expectation & $9.5 \pm 0.86$ & $9.07 \pm 0.74$ & 0.192 \\
Functional Activities & $33.63 \pm 4.57$ & $31.37 \pm 4.8$ & 0.101 \\
Symptoms & $14.3 \pm 1.76$ & $14.5 \pm 1.68$ & 0.587 \\
Range of Motion & $87.5 \pm 6.92$ & $92.33 \pm 6.26$ & 0.316 \\
\hline \multicolumn{4}{c}{ Preoperative mean knee society score } \\
\hline The 2011 KSS categories & CR & PS & P Value \\
\hline Objective Knee Indicators & $71.17 \pm 3.78$ & $73.13 \pm 2.29$ & 0.018 \\
Satisfaction & $21.6 \pm 4.34$ & $21.6 \pm 3.34$ & 1.000 \\
Expectation & $9.93 \pm 1.17$ & $9.57 \pm 0.97$ & 0.192 \\
Functional Activities & $69.23 \pm 6.83$ & $66.37 \pm 6.48$ & 0.101 \\
Symptoms & $7.23 \pm 1.28$ & $7.03 \pm 1.54$ & 0.654 \\
Range of Motion & $104.2 \pm 19.58$ & $114.67 \pm 8.9$ & 0.010 \\
\hline
\end{tabular}
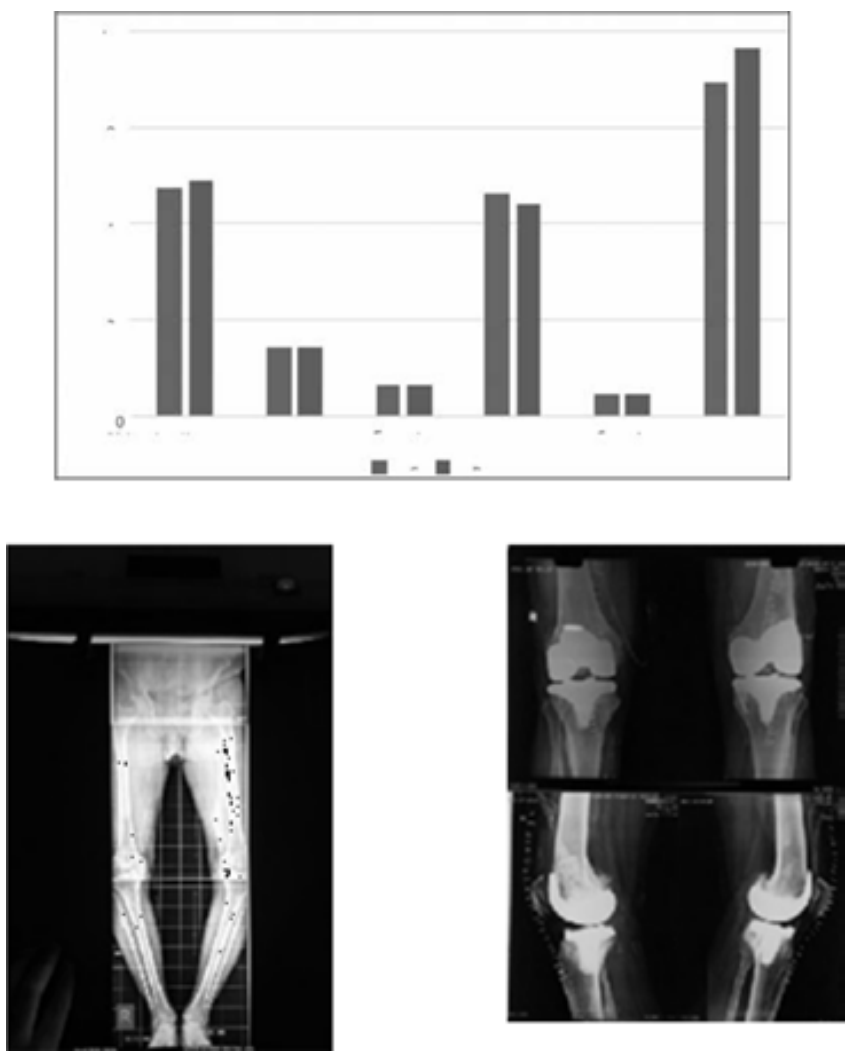

Fig 1. Postoperative Knee Society Score

Preoperative and Postoperative X-ray of case operated with PS prosthesis
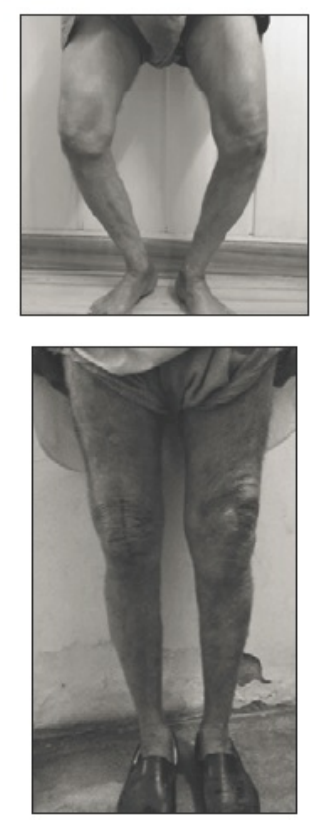

Fig 2. Case Operated With B/L PS
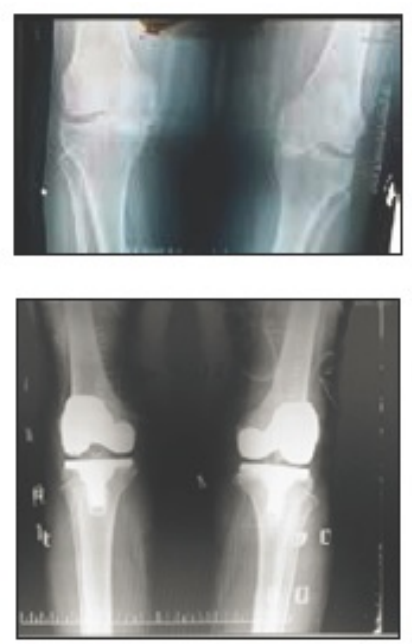

Fig 3. Preoperativeand Postoperative $x$-ray of case operated with $B / L$ CR prosthesis

\section{Discussion}

As patient expectations following knee arthroplasty increases, knee arthroplasty research is increasingly focused on clinically important differences in patient functional outcomes but still there is controversy whether the Cruciate retaining or posterior stabilised prosthesis designs have superior outcomes over each other. Proponents of cruciate-retaining designs believe that it is important to retain as much of the original anatomy as possible, and that the PCL can continue 


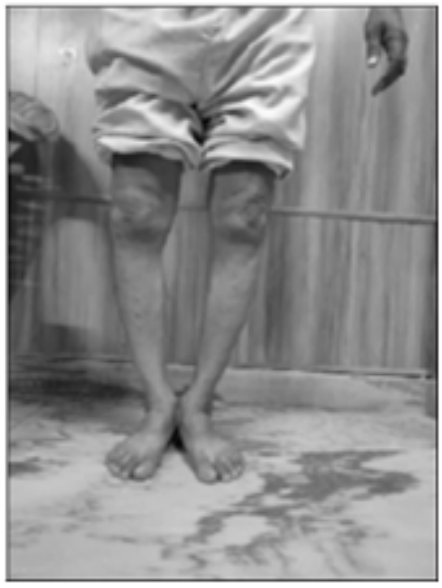

Fig 4.

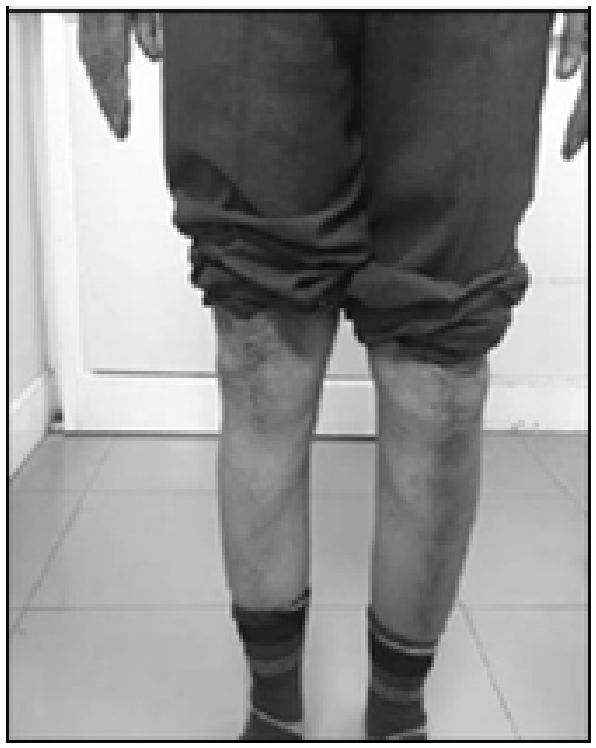

Fig 5. Case Operated With B/LCR

to stabilize the knee during flexion. The posterior-stabilized designs utilize a tibial post and femoral cam to substitute for the PCL, which allows femoral rollback and attempts to prevent anterior movement of the femur.

After TKA treatment, ROM of the operated knee is a very important index when evaluating the effect. Most of the previous report found that post-operative Range of motion was better in PSTKA. In our study, mean ROM for CR pros thesis preoperatively and postoperatively was 87.5 and 104.67 respectively where as it was 92.33 and 114.67 for PS. These results are comparable with the studies done by $\mathrm{N} \mathrm{Li}$ et al ${ }^{(9)}$, Matsumoto et $\mathrm{al}^{(10)}$, Yagishita et $\mathrm{al}^{(11)}$ Berick et al $^{(12)}$; where they also indicated more significant improvement in ROM in PS TKA as compared to CR TKA. The mean objective knee score in the present study for CR and PS prosthesis was 33.17 and 39.1 respectively which got improved to 71.17 and 73.13 which indicated significant improvement but in contrast to Suthar RK et $\mathrm{al}^{(13)}$, Longo UG et $\mathrm{al}^{(14)}$ study; favours PS prosthesis with better objective knee score results.

The mean knee society score for pain in our study was 14.3 and 7.23 preoperatively and postoperatively respectively for CR prosthesis whereas it was 14.5 and 7.03 pre and postoperatively for PS TKA. These results are comparable to $\mathrm{N}$ Li et al ${ }^{(9)}$, Wang CJ et al ${ }^{(15)}$, Verra et al ${ }^{(16)}$ study where they also indicated no statistically significant difference.

The question of function in cruciate retaining and posterior stabilized knee replacements has been addressed in several studies. The study by Frank R. kolisek et al ${ }^{(17)} 18$, $\mathrm{R}$ chaudary et $\mathrm{al}^{(18)}$, EA unkar et $\mathrm{al}^{(19)}$, Harato et $\mathrm{al}^{(20)}$ concluded insignificant difference in knee functional score between CR and PS TKA at follow up in patients operated with these two prosthetic types. In our study the mean postoperative knee function score for CR group was 69.23 and for PS group was 66.37 with $p$ value of 0.101 which also concluded insignificant difference between both groups, despite the superior range of motion in PS TKA what still remains uncertain is whether these small increases in knee motion in PS prostheses represent clinically relevant improvements that are recognized by patients. Similarly, there was insignificant difference between CR and PS Total knee arthroplasty regarding patient satisfaction and expectation. Thus, the differences in ROM and knee flexion that have been found to be statistically significant in favour of PS group may not be significant to the patient.

The PCL has been shown to provide approximately $95 \%$ of the total restraint to posterior translation of the tibia on the femur, maximally taut at full flexion. Thus, CR TKA is advantageous over PS TKA as it retain the PCL and also being the bone conserving technique as compared to PS TKA where more of intercondylar bone stock and femoral condyles are sacrificed for prostheis. PCL, if found to be intact in an arthritic knee can be balanced. But many authors argue that the PCL is diseased with various form of arthritis and contracture and is difficult to balance reproducibly and also PCL in some cases contributes to the flexion and varus deformity; PCL being the medial stabilising structure. When medial structures are subsequently released to balance lax lateral structures, the PCL is usually too tight relative to these structures and must be released to some extent to balance the knee but there exists the possibility for late anteroposterior instability if the PCL stretches over time. One of the biggest drawback of the CR prosthesis is late rupture of PCL, a issue which can be addressed by using deep dish insert in CR TKA. Cruciate retaining implants rely on the intact PCL, which becomes taut in deep flexion, preventing anterior femoral translation and producing femoral roll back However, recent kinematic studies have thrown doubt over this mechanism 
and have shown paradoxical anterior movement of the femoral condyles during high-flexion with higher peak femorotibial contact stress. ${ }^{(21,22)}$ This may be are result of difficulty in adequately balancing the PCL or of pathological PCL function, as it may be involved in the degenerative disease process and no longer functions like a "normal" PCL, following knee replacement. In contrast, cruciate substituting knee replacement relies on replacement of the PCL with a tibial polyethylene post, which articulates with a CAM between the femoral condyles. This CAM/post articulation in deep flexion prevents anterior femoral translation and mechanically reproduces femoral rollback. Kinematic studies have demonstrated that this mechanical rollback creates a smoother flexion arc and also a superior range of deep flexion

Our study is potentially important and should guide surgeons when selecting which prosthesis to implant in their patients. However, further research, into this field especially kinematic and anatomic study is necessary and only through a large prospective randomized controlled trial would it be possible to truly determine which implant is superior in terms of long-term functional outcome.

\section{Conclusion}

There are surgeons who exclusively do PS TKA in all the cases but we found that with PCL recession and soft tissue balancing and use of deep dish insert, CR TKA can be done even in cases with varus deformity. In the end, we suggest that if the contractures are significant, and the PCL is adding to the deformity, it should be excised and posterior stabilized prosthesis should be used. On the other hand, if the PCL seems adequate and does not hinder the surgical procedure, and seems to be adding to the stability, it should preferably be retained, as it has significant stabilising function.

\section{References}

1) Mihalko MW. Arthroplasty of the knee. In: Azar FM, et al., editors. Campbell's operative orthopaedics. 13;Philadelphia. Elsevier. 2017;p. 397.

2) Andriacchi TP, Galante JO, Fermier RW. The influence of total knee replacement design on walking and stair climbing. J Bone Joint Surg Am. 1982;64:1328-1335.

3) Kelman JG, Biden NE, Wyatt PM, Ritter AM, Colwel WC. Gait laboratory analysis of a posterior cruciate-sparing total knee arthroplasty in stair ascent and descent. Clinical Orthopaedics and Related Research. 1989;(248). Available from: https://dx.doi.org/10.1097/ 00003086-198911000-00006.

4) Maloney JW, Schurman JD. The effects of implant design on range of motion after total knee arthroplasty. Clinical Orthopaedics and Related Research. 1992;278:147-152. Available from: https://dx.doi.org/ 10.1097/00003086-199205000-00024.

5) Long WJ, Scuderi GR. Varus and Valgus deformities. In: Lotke PA, Lonner LH, et al., editors. Knee Arthroplasty;vol. 3. Newyork. Lippincott Williams \&Wilkins. 2009;p. 111.

6) Maruyama S, Yoshiya S, Matsui N, Kuroda R, Kurosaka M. Functional comparison of posterior cruciate-retaining versus posterior stabilized total knee arthroplasty. The Journal of Arthroplasty. 2004;19(3):349-353. Available from: https://dx.doi.org/10.1016/j.arth.2003.09.010.
7) Jacobs WC, Clement DJ, Wymenga AB. Retention ver- sus sacrifice of the posterior cruciate ligament in total knee replacement for treatment of osteoarthriti and rheumatoid arthritis. Cochrane Database Syst Rev. 2005;(4).

8) Seon JK, Park JK, Shin YJ, et al. Comparisons of kinematics and range of motion in high-flexion total knee arthroplasty: cruciate retaining vs.substituting designs. Knee Surg Sports Traumatol Arthrosc. 2011;19(12):2016-2022.

9) Li N, Tan Y, Deng Y. Posterior cruciate-retaining versus posterior stabilized total knee arthroplasty: a meta-analysis of randomized controlled trials. Knee Surg Sports Traumatol Arthrosc. 2014;22(3):556564.

10) Matsumoto T, Muratsu H, Kubo S, Matsushita T, Kurosaka M, Kuroda R. Intraoperative soft tissue balance reflects minimum 5-Year midterm outcomes in cruciate-retaining and posterior-stabilized total knee arthroplasty. The Journal of Arthroplasty. 2012;27(9):1723-1730. Available from: https://dx.doi.org/10.1016/j.arth.2012.02.020.

11) Yagishita K, Muneta T, Ju YJ, Morito T, Yamazaki J, Sekiya I. High-flex posterior cruciate-retaining vs posterior cruciate-substituting designs in simultaneous bilateral total knee arthroplasty. The Journal of Arthroplasty. 2012;27:368-374. Available from: https://dx.doi.org/10. 1016/j.arth.2011.05.008.

12) Bercik MJ, Joshi A, Parvizi J. Posterior cruciate -retaining versus posterior -stabilized total knee arthroplasty: A Meta analysis. J arthroplasty. 2013;28:439-444.

13) Suthar KR, Parekh RD, Mistry BS. Cruciate retaining versus posterior-stabilized total knee arthroplasty: a short-term comparative study. International Journal of Research in Orthopaedics. 2018;4:726726. Available from: https://dx.doi.org/10.18203/issn.2455-4510. intjresorthop20183671.

14) Longo UG, Mannering N, D’Andrea V, Locher J, Salvatore G, Denaro V, et al. Outcomes of Posterior-Stabilized Compared with CruciateRetaining Total Knee Arthroplasty. The Journal of Knee Surgery. 2018;31(04):321-340. Available from: https://dx.doi.org/10.1055/s0037-1603902.

15) Jiang C, Liu Z, Wang Y, Bian Y. Posterior Cruciate Ligament Retention versus Posterior Stabilization for Total Knee Arthroplasty: A Meta-Analysis. PLOS / ONE TENTH ANNIVERSARY. 2016;11(1). Available from: https://Journals.Plos.org/Plosone/article.DOI.org/10. 1371/journal.pone.0147865.

16) Verra CW, van den Boom GHL, Jacobs CHW, Schoones WJ, Wymenga BA, Nelissen GHHR. Similar outcome after retention or sacrifice of the posterior cruciate ligament in total knee arthroplasty. Acta Orthopaedica. 2015;86(2):195-201. Available from: https://dx.doi.org/ $10.3109 / 17453674.2014 .973329$.

17) Kolisek FR, Mcgrath MS, Marker DR, Jessup N, Seller TM, Mont MA. Posterior -stabilzed versus posterior crucite ligament -retaining Total knee Arthroplasty. Iowa orthop J2009;29:23-37.

18) Chaudhary R, Beaupré LA, Johnston DWC. Knee Range of Motion During the First Two Years After Use of Posterior Cruciate-Stabilizing or Posterior Cruciate-Retaining Total Knee Prostheses. The Journal of Bone and Joint Surgery-American Volume. 2008;90(12):2579-2586. Available from: https://dx.doi.org/10.2106/jbjs.g.00995.

19) Ünkar EA, Öztürkmen Y, Şükür E, Çarkçı E, Mert M. Posterior cruciate-retaining versus posterior-stabilized total knee arthroplasty for osteoarthritis with severe varus deformity. Acta Orthopaedica et Traumatologica Turcica. 2017;51(2):95-99. Available from: https://dx. doi.org/10.1016/j.aott.2016.12.008.

20) Harato K, Bourne RB, Victor J. Midterm comparison of posterior cruciate-retaining versus-substituting total knee arthroplasty using the Genesis II prosthesis. A multicentre prospective randomized clinical trial. Knee. 2008;15(3):217-221.

21) Fantozzi S, Catani F, Ensini A, Leardini A, Giannini S. Femoral rollback of cruciate-retaining and posterior-stabilized total knee replacements: In vivo fluoroscopic analysis during activities of daily living. Journal of Orthopaedic Research. 2006;24(12):2222-2229. Available from: https: //dx.doi.org/10.1002/jor.20306. 
22) Stiehl JB, Komistek RD, Cloutier JM. The cruciate ligaments in total knee arthroplasty: a kinematic analysis of 2 total knee arthroplasties.
Arthroplasty. 2000;15(5):545-550. 